${ }^{\circledR}$ Entomologica Fennica. 18 April 1997

\title{
Additional records and new synonyms of Cicindelidae and Carabidae (Coleoptera) from the Island of Sakhalin in the Russian Far East
}

\author{
German Sh. Lafer, Anders N. Nilsson \& Sergey K. Kholin
}

Lafer, G. Sh., Nilsson, A. N. \& Kholin, S. K. 1997: Additional records and new synonyms of Cicindelidae and Carabidae (Coleoptera) from the Island of Sakhalin in the Russian Far East. — Entomol. Fennica 8: 13-17.

Faunistic records of three cicindelid and 47 carabid beetle species are given for the Island of Sakhalin in the Russian Far East. Most records refer to a material collected in 1993 and 1994 in SW Sakhalin by the two junior authors. The following five carabid species are here reported from Sakhalin for the first time: Bembidion chloropus Bates, Pterostichus neglectus A. Morawitz, Agonum jankowskii Lafer, A. piceum (Linnaeus) and Microlestes minutulus (Goeze). The gross distributions of all mentioned species are given, with special emphasis on the Far East. The following syn. nov. are given: Pterostichus (Euryperis) eximius A. Morawitz, 1862 (= crassiceps A. Morawitz, 1862; = rudnicus Jedlicka, 1938; = obliquebasalis Jedlicka, 1962; = prochazkorum Jedlicka, 1967; = sakhalinensis Kirschenhofer, 1985).

German Sh. Lafer \& Sergey K. Kholin, Laboratory of Entomology, Institute of Biology and Soil Sciences, Vladivostok 22, 690022, Russia

Anders N. Nilsson, Department of Animal Ecology, University of Umeå, S-901 87 Umeå, Sweden

Received 30 October 1995, accepted 21 March 1996

\section{Introduction}

Sakhalin, that is one of the largest islands of the North Pacific, is situated along the eastern coast of Asia between the mouth of the Amur River and Hokkaido, between $141^{\circ} 38^{\prime}$ and $144^{\circ} 55^{\prime} \mathrm{N}$. It is about $950 \mathrm{~km}$ long and covers a total area of $76400 \mathrm{~km}^{2}$. In the north, Sakhalin is separated from the continent by the $7.5-\mathrm{km}$ wide Nevelskoy Channel and in the south from Hokkaido by the 37.5-km wide La Pérouse Strait.

About 200 species of Cicindelidae and Carabidae are known from the Island of Sakhalin (Lafer, unpublished). Lafer $(1989,1992)$ listed the known records of most species, except the two speciesrich genera Bembidion and Pterostichus. In this paper, we report on the material collected on South Sakhalin chiefly by the two junior authors in 1993 and 1994. This material includes 236 specimens representing fifty species, of which five are new for the Sakhalin fauna of ground beetles (Bembidion chloropus, Pterostichus neglectus, Agonum jankowskii, A. piceum and Microlestes minutulus). The poor faunistic knowledge of the fauna of this island in literature has motivated the publication of all species records. 


\section{Material and methods}

All listed localities belong to the Kholmsk administrative district in the southwest part of Sakhalin (see map in Nilsson \& Kholin 1994). The following localities were studied: (1) Pionery - $22 \mathrm{~km} \mathrm{~N}$ of Kholmsk, (2) Kostromskoye - $25 \mathrm{~km}$ $\mathrm{N}$ of Kholmsk, (3) Kholmsk City, and (4) Pyatirechye $15 \mathrm{~km}$ E of Kholmsk. Collecting was chiefly performed in the following biotope types: coastal sand dunes (localities 2 and 3), Carex marshes and bogs ( 2 and 3), mixed deciduous forests on the coastal hills (1-3), and river valleys with broadleaved forest (3 and 4).

Most specimens were collected by hand, in wet habitats often in combination with pressing down littoral vegetation and moss under the water or pouring water on minerogenic shores. Pitfall traps were used in Pionery and Kostromskoye. The material is deposited in the collection of the Swedish Museum of Natural History, Stockholm, and a few specimens are placed in the collection of the Institute of Biology and Soil Science, Vladivostok (IBSS). The records cited without reference to any other collector refer to the material collected by the two junior authors. The number of specimens are given within parentheses. All beetles were identified by the senior author. For each species also the gross geographical distribution (following Lafer 1989 and unpublished) is given.

\section{List of species}

\subsection{Cicindelidae}

Cicindela (Cicindela) transbaicalica Motschulsky, 1844. Pionery, 22-26.VI.1993 (5); Kostromskoye, 18-26.VI.1993 (4). - SE Siberia, S Russian Far East excluding S Kuriles, Mongolia, China, Korea, Japan. In Sakhalin, this species is so far known only from the Kholmsk district.

Cicindela (Cicindela) sachalinensis A. Morawitz, 1862. —Pionery, 22-26.VI.1993 (2). — S Russian Far East, Sakhalin, S Kuriles (Shikotan, Kunashir, Iturup), China, Japan (Hokkaido, Honshu).

Cicindela (Cicindela) sylvatica Linnaeus, 1758. - Pionery, 9.VIII.1994 (1); Kostromskoye, 19.VI.1993 (1). The forest zone of Europe and Siberia, S Russian Far East excluding most of Primorye and the Kuriles, Sakhalin.

\subsection{Carabidae}

Nebria (Boreonebria) subdilatata Motschulsky, 1845. Kostromskoye, 23.VI.1993 (2), — Altai and Sayan Mountains, E Siberia and Russian Far East to Kamtchatka, Sakhalin, S Kuriles (Kunashir), Korea.

Nebria (Reductonebria) ochotica R. F. Sahlberg, 1844. - Pyatirechye, 25.VI.1993 (2), — E Siberia, Russian Far East, Sakhalin, Kurile Islands, Japan (Hokkaido), Korea.
Carabus (Adelocarabus) arboreus Lewis, 1882. - Pionery, 22-26.VI.1993 (1). — Japan (Hokkaido, N Honshu), Sakhalin, S Kuriles (Kunashir).

Carabus (Carabus) granulatus Linnaeus, 1758. Pionery, 22-26.VI.1993 (3). - The forest zone of Eurasia, Sakhalin, S Kuriles (Shikotan, Kunashir, Iturup), Japan (Hokkaido, N Honshu), Korea, E China.

Carabus (Homoeocarabus) maeander Fischer von Waldheim, 1820. - Kostromskoye, 23.VI.1993 (1). - E Siberia from Irkutsk Region to Kamtchatka, S Russian Far East, Sakhalin, S Kuriles (Kunashir), Japan (Hokkaido), North America.

Elaphrus (Elaphrus) riparius (Linnaeus, 1758). - Kostromskoye, 23.VI.1993 (3). - Europe, Siberia, Russian Far East, Sakhalin, S Kuriles (Kunashir), Japan, NE China, North America.

Elaphrus (Neoelaphrus) sibiricus Motschulsky, 1845. - Pionery, 22-23.VI.1993 (7). - Siberia, S Russian Far East, Sakhalin, S Kuriles (Kunashir, Shikotan), Korea, Japan.

Loricera pilicornis (Fabricius, 1775). - Pionery, 22 26.VI.1993 (4); Kostromskoye, 23.VI.1993 (2). - Europe, Siberia, Russian Far East, Sakhalin, Kurile Islands, Japan (Hokkaido), North America.

Diplous depressus Gebler, 1830. - Pyatirechye, 25.VI.1993 (5). - Siberia to Magadan Region and Primorye, Sakhalin, NE China, Korea, Japan.

Bembidion (Bembidion) paediscum Bates, 1883. Pionery, 22-26.VI.1993 (1). — Sakhalin, S Kuriles (Shikotan, Kunashir, Iturup), Japan (Hokkaido, Honshu).

Bembidion (Chrysobracteon) conicolle Motschulsky, 1845. - Kostromskoye, 23.VI.1993 (1). - Siberia, Russian Far East, Sakhalin, Japan (Hokkaido).

Bembidion (Cylindrobracteon) chloropus Bates, 1883. - Pionery, 22-26.VI.1993 (8); Novoaleksandrovsk, 12.VII.1993 (1) V. N. Kuznetzov (IBSS), - The first records from Sakhalin. S Primorye, Sakhalin, S Kuriles (Iturup, Kunashir), Japan (Hokkaido, Honshu), Korea, NE China.

Bembidion (Diplocampa) prostratum Motschulsky, 1842. - Pionery, 22-26.VI.1993 (12), 18.VIII.1994 (1); Kostromskoye, 18-26.VI.1993 (10), 15.VIII.1994(1); Pyatirechye, 25.VI.1993 (4). - E Siberia from Baikal to Kamtchatka and S Russian Far East, Sakhalin, S Kuriles (Kunashir), Japan (Hokkaido), Mongolia.

Bembidion (Eupetodromus) sibiricum Dejean, 1831 (= inouyei Habu). — Kholmsk, 21.VI.1993 (6); Pionery, 22 23.VI.1993 (4). - Kazakhstan, Altai, Siberia to Kamtchatka and S Russian Far East, Sakhalin, Japan (Hokkaido).

Bembidion (Notaphus) obliquum fasciatum Motschulsky, 1842. - Kholmsk, 21.VI.1993 (1); Pionery, 23.VI.1993 (1); Kostromskoye, 18-26.VI.1993 (9). - E Siberia from Irkutsk Region to Kamtchatka and S Russian Far East, Sakhalin, S Kuriles (Kunashir).

Bembidion (Peryphus) ?pohlai Kirschenhofer, 1984 (= sp. pr. captivorum Netolitsky). - Pionery, 22-26.VI.1993 (2). - Sakhalin, S Kuriles (Kunashir, Iturup), Japan (Hokkaido).

Bembidion (Peryphus) dolorosum Motschulsky, 1860. - Kostromskoye, 18-26.VI.1993 (1); Pyatirechye, 
25.VI.1993 (10). - Sakhalin, S Kuriles (Kunashir, Iturup, Shikotan, Urup), Japan (Hokkaido).

Bembidion (Peryphus) morawitzi Csiki, 1928. - Kostromskoye, 15.VIII.1994 (1). Additional previously unpublished material: "Saghalien Cent. Exp. Sta." Takinosawa, 27.IX.1929 (1) K. Tamanuki; Siritori (Makarov), 7.V.1933 (1), 18.VI.1933 (2), 28.VI.1933 (1) M. Yasikura (IBSS). — S Russian Far East, Sakhalin, S Kuriles (Kunashir), NE China, Korea, Japan.

Bembidion (Plataphodes) tetraporum Bates, 1883. Kostromskoye, 23.VI. 1993 (3); Pyatirechye, 25.VI.1993 (5). —S Russian Far East, Sakhalin, S Kuriles (Shikotan, Kunashir, Iturup), Japan (Hokkaido, Honshu).

Poecilus (Macropoecilus) fortipes Chaudoir, 1850. Kostromskoye, 18-26.VI.1993 (1); Pionery, 22-26.VI.1993 (2). - Siberia to Kamtchatka, Sakhalin, S Kuriles (Kunashir), Mongolia, China, Korea, Japan.

Pterostichus (Argutor) diligens (Sturm, 1824). - Pionery, 22-26.VI.1993 (2). - Europe, Middle Asia, Siberia, Kamtchatka, Sakhalin, S Kuriles (Shikotan, Kunashir, Iturup).

Pterostichus (Badistrinus) neglectus A. Morawitz, 1862. — Pionery, 22-26.VI.1993 (3); Kostromskoye, 19.VI.1993 (1). - The first record from Sakhalin. S Russian Far East, Sakhalin, S Kuriles (Kunashir), NE China, Japan (Hokkaido).

Pterostichus (Bothriopterus) adstrictus Eschscholz, 1823. - Pionery, 22-26.VI.1993 (5). - N Europe, Siberia, Russian Far East, Sakhalin, Kurile Islands, Korea, Japan, North America.

Pterostichus (Cryobius) kurasawai Tanaka, 1958. Pionery, 22-26.VI.1993 (1). - This species is endemic to Sakhalin.

Pterostichus (Euryperis) eximius A. Morawitz, 1862. - Pionery, 22-26.VI.1993 (1). — E Siberia from the Baikal and W Yakutia to Magadan Region, Chukotka, Koryakia in the north and the northern Sikhote-Alin, Sakhalin, Mongolia.

Pterostichus (Eurythoracana) haptoderoides Tschitscherine, 1888. - Pionery, 22-26.VI.1993 (1). — E Siberia from Baikal to Khabarovsk and Primorye Territories, Sakhalin, S Kuriles (Kunashir, Iturup), Korea, China, Japan (Hokkaido, Honshu).

Pterostichus (Melanius) nigrita (Paykull, 1790), Kholmsk, 21.VI.1993 (2); Pionery, 22-26.VI.1993 (17); Kostromskoye, 18-26.VI.1993 (4). —Europe, Minor Asia, Kazakhstan, Siberia, Russian Far East, Sakhalin, S Kuriles (Kunashir, Iturup), Mongolia, China, Korea, Japan.

Pterostichus (Steropus) alacer A. Morawitz, 1862. Kholmsk, 21.VI.1993 (1). — S Russian Far East excluding $S$ Kuriles, Sakhalin.

Agonum (Agonum) dolens (C. Sahlberg, 1827). Kholmsk, 21.VI.1993 (5). - N \& C Europe, Kazakhstan, Siberia, Russian Far East, Sakhalin, Korea, NE China, Japan (Hokkaido, N Honshu).

Agonum (Agonum) impressum (Panzer, 1797). - Pionery, 22-26.VI.1993 (4). - Europe, Kazakhstan, Siberia, Russian Far East, Sakhalin, S Kuriles (Shikotan, Kunashir, Iturup), Mongolia, NE China, Korea, Japan.
Agonum (Agonum) jankowskii Lafer, 1992. - Kostromskoye, 19.VI.1993 (6). - The first record from Sakhalin. S Russian Far East, S Sakhalin.

Agonum (Agonum) sculptipes Bates, 1883. - Pionery, 22-26.VI.1993 (1); Kostromskoye, 18-26.VI.1993 (1). SE Siberia from Baikal to Khabarovsk and Primorye Territories, Sakhalin, Mongolia, NE China, Korea.

Agonum (Eucolpodes) japonicum (Motschulsky, 1860). - Pionery, 22-26.VI. 1993 (3); Kholmsk, 21. VI.1993 (10). - Sakhalin, S Kuriles (Shikotan, Kunashir, Iturup), Japan, Korea.

Agonum(Europhilus) gracile Sturm, 1824. - Kostromskoye, 15.VIII. 1994 (1). - - Europe, S Siberia to Primorye, Sakhalin, Japan (Hokkaido).

Agonum (Europhilus) piceum (Linnaeus, 1758). Kholmsk, 21.VI.1993 (1); Pionery, 22-26.VI.1993 (6). The first record from Sakhalin. Europe, Siberia to Khabarovsk and Primorye Territories, Sakhalin, S Kuriles (Shikotan, Kunashir), NE China, Korea, Japan (Hokkaido).

Agonum (Europhilus) subtruncatum Motschulsky, 1860. - Pionery, 22-26.VI. 1993 (4). - Russian Far East, Sakhalin, S Kuriles (Shikotan, Kunashir, Iturup), Japan (Hokkaido).

Agonum(Europhilus) thoreyi Dejean, 1828. - Pionery, 22-26.VI.1993 (1); Kostromskoye, 18-26.VI.1993 (1). Europe, Siberia to Khabarovsk and Primorye Territories, Kamtchatka, Sakhalin, S Kuriles (Kunashir), Japan (Hokkaido, Honshu), North America.

Agonum (Platynus) assimilis (Paykull, 1790). - Pyatirechye, 25.VI.1993 (1). - Europe, Minor Asia, Siberia to Primorye, Sakhalin, Mongolia, Japan.

Amara (Amara) communis (Panzer, 1797). - Pionery, 22-26.VI.1993 (1); Kostromskoye, 18-26.VI.1993 (4). Europe, Middle Asia, Kazakhstan, Siberia to Kamtchatka and Primorye Territory, Sakhalin, S Kuriles (Kunashir, Iturup), NE China.

Amara (Amara) similata chalcites Dejean, 1828. Kholmsk, 21.VI.1993 (1); Pionery, 22-26.VI.1993 (1); Kostromskoye, 18-26.VI.1993 (2). — Europe, Middle Asia, Kazakhstan, Siberia to Kamtchatka and Khabarovsk and Primorye Territories, Sakhalin, S Kuriles (Kunashir, Iturup), NE China, Korea, Japan.

Amara (Amara) ussuriensis Lutshnik, 1935. - Pionery, 22-26.VI.1993 (1). - SE Siberia from Yenisey River to Kamtchatka and Primorye, Sakhalin, S Kuriles (Kunashir, Iturup), Korea, N China, Mongolia.

Amara (Zezea) plebeja (Gyllenhal, 1810). — Kostromskoye, 18-26.VI.1993 (1). — Europe, Siberia to Yakutia and Primorye, Sakhalin, S Kuriles (Kunashir, Iturup), Japan (Hokkaido), Korea, NE China.

Anisodactylus signatus (Panzer, 1797). - Pionery, 2226.VI.1993 (2). - Europe, Middle Asia, Iran, Kazakhstan, S Siberia to Khabarovsk and Primorye Territories, Sakhalin, S Kuriles (Kunashir, Iturup), Mongolia, China, Korea, Japan.

Stenolophus (Stenolophus) propinquus A. Morawitz, 1862. - Pionery, 23.VI.1993 (1). - S Russian Far East, Sakhalin, S Kuriles (Shikotan, Kunashir), Korea, NE \& E China, Japan. 
Chlaenius (Chlaenius) insularis Kryzhanovskij, 1973. - Pionery, 22-26.VI.1993 (3); Kostromskoye, 1826.VI.1993 (1), - Sakhalin, S Kuriles (Kunashir), Japan (Hokkaido).

Demetrias amurensis Motschulsky, 1860. - Pionery, 22-26.VI.1993 (3); Kostromskoye, 19.VI.1993 (1). — S Russian Far East, Sakhalin, S Kuriles (Shikotan, Kunashir), Korea, NE China, Japan (Hokkaido), Korea, NE China.

Microlestes minutulus (Goeze, 1777). - Kostromskoye, 18-26.VI.1993 (4). - The first record from Sakhalin. Europe, N Africa, Middle Asia, Kazakhstan, Siberia to Khabarovsk and Primorye Territories, Sakhalin, NE China, Japan (Hokkaido, Honshu).

\section{New synonyms}

Pterostichus (Euryperis) eximius A. Morawitz, 1862

= Pterostichus crassiceps A. Morawitz, 1862, syn. nov.

= Pterostichus rudnicus Jedlicka, 1938, syn. nov. = Pterostichus obliquebasalis Jedlicka, 1962, syn. nov.

= Pterostichus prochazkorum Jedlicka, 1967, syn. nov.

= Pterostichus sakhalinensis Kirschenhofer, 1985, syn. nov.

The previously unpublished synonymy of $P$. obliquebasalis Jedlicka (holotype male from "Borochojewa Transbaikal") and P. prochazkorum Jedlicka (holotype female from Ulanbator, Mongolia) with P. eximius A. Morawitz have been established by G. Shilenkov (Irkutsk) in 1977 and is now confirmed by the senior author. The other synonyms have been established by the senior author and are here published for the first time. The types of A. Morawitz have been studied in the Zoological Institute of St. Petersburg in 1973. $P$. eximius A. Morawitz was described from two syntypes ("Zagan-olui" and "Tschindansk") from Transbaikalien (Tchita Region). In the same work Morawitz (1862) described this species also as Pterostichus (Steropus) crassiceps, based on a female from De Castries Bay (Khabarovsk Territory), located on the continent opposite to Sakhalin.

$P$. rudnicus Jedlicka was described from two specimens labelled "Sutschanski-Rudnik Ussuri". These specimens are identical with $P$. eximius A. Morawitz but no one have collected this species in Primorye again. Probably the types of $P$. rud- nicus are mislabelled, and may more likely have been obtained by Dr. K. Mandl in Transbaikalien and not in the Ussuri Region.

Kirschenhofer (1985) described $P$. eximius from a male from Mt. Tchekov on Sakhalin under the name Pterostichus (Steropus) sakhalinensis. The detailed original description, including illustrations of male genitalia and photo of the holotype, does not leave any doubt that $P$. sakhalinensis is identical with $P$. eximius A. Morawitz. The series of this species from Sakhalin in coll. IBSS includes more than 100 specimens.

The majority of the above-mentioned species were first described in the subgenus Steropus Stephens sensu auct. (type species $P$. aethiops Panzer). However, the aedeagi of the true Steropus species have the characteristic apex bent upwards and towards the right. Moreover, the last abdominal segments are modified and the pronotal hind angles lack latero-basal carinae. In contrast, $P$. eximius is characterized by the flat subtriangular penis apex, a pronotum with latero-basal carinae present at hind angles, and simple last abdominal sternites as in the subgenus Euryperis Motschulsky.

Acknowledgements. We thank the Swedish Museum of Natural History, Stockholm, for a travelling grant to A. N. Nilsson. Dr. Z. Mlynar of the Prague Museum, on the request of the senior author, kindly sent the types of Jedlicka's species $P$. rudnicus, $P$. obliquebasalis and $P$. prochazkorum to Vladivostok.

\section{References}

Jedlicka, A. 1938: Versuch einer Bestimmungstabelle der mir bekannten Pterostichus-Arten aus Ostasien. Praha, 11 pp.

Jedlicka, A. 1962: Monographie des Tribus Pterostichini aus Ostasien (Pterostichi, Trigonotomi, Myadi) (Coleoptera, Carabidae). —Ent. Abhandl. Staatl. Mus. Tierkunde Dresden 26(21): 177-346.

Jedlicka, A. 1967: Ergebnisse der 1. mongolisch-tschechoslowakischen entomologisch-botanischen Expedition in der Mongolei. 4. Coleoptera-Carabidae. - Acta faun. ent. Mus. Nat., Pragae 12: 103-114.

Kirschenhofer, E. 1985: Zwei neue Pterostichus (Steropus) sakhalinensis sp. n. und Pterostichus (Lyperopherus) wellschmiedi sp. n. (Coleoptera, Carabidae). - Entomofauna 6: 221-229.

Lafer, G. Sh. 1989: Family Carabidae - Ground Beetles. Keys to the insects of the Far East of the USSR 3(1): 71-222. (In Russian.) 
Lafer, G. Sh. 1992: Family Carabidae - Ground Beetles. Keys to the insects of the Far East of the USSR 3(2): 602-621. (In Russian.)

Morawitz, A. 1862: Vorlaufige Diagnosen neuer Coleopteren aus Südost Sibirien. - Melanges biologiques tires du Bulletin de L'Academie Imperiale des Sciences de St.-Petersbourg 4(2): 180-228.

Nilsson, A. N. \& Kholin, S. K. 1994: The diving beetles (Coleoptera, Dytiscidae) of Sakhalin - an annotated checklist. - Entomol. Tidskr. 115: 143-156. 